EPJ manuscript No.

(will be inserted by the editor)

\title{
Kaon condensation in neutron star using relativistic mean field models
}

\author{
S. W. Hong ${ }^{1}$, C. H. Hyun ${ }^{1}$, and C. Y. Ryu ${ }^{2}$ \\ 1 Department of Physics and Institute of Basic Science, Sungkyunkwan Univ., Suwon 440-746, Republic of Korea \\ 2 Research Center for Nuclear Physics (RCNP), Ibaraki, Osaka 567-0047, Japan
}

Received: date / Revised version: date

\begin{abstract}
We use the modified quark-meson coupling and the quantum hadrodynamics models to study the properties of neutron star. Coupling constants of both models are adjusted to reproduce the same saturation properties. The onset of kaon condensation in neutron star matter is studied in detail over a wide range of kaon optical potential values. Once the kaon condensation takes place, the population of kaons increases very rapidly, and kaons become the dominant component, possibly making the neutron star matter a kaonic matter if the kaon optical potential is large.

PACS. 97.60.Jd Neutron stars - 14.20.Jn Strange particles
\end{abstract}

\section{Introduction}

Observations of the masses of binary pulsars [1, which are candidates of neutron stars indicate that the maximum mass of neutron stars are roughly around $1.5 M_{\odot}$. While the mass of a neutron star with only neutrons and protons is estimated to be about $2 M_{\odot}$, exotic degrees of freedom 2, 3, 4, other than the nucleons seem to reduce the maximum mass of a neutron star close to the observed values, which implies that the exotic degrees of freedom such as creation of hyperons, Bose-Einstein condensation, strange matter, and quark deconfinement seem to be needed to reproduce the observed masses of neutron stars.

In this work, we consider the strangeness degrees of freedom by including hyperon creation and kaon condensation in the neutron star matter. The equation of state (EoS) of dense nuclear matter is sensitive to the in-medium interaction of the hyperons and kaons. Recently, the magnitudes of the kaon-nucleus potential in matter have attracted much attention. Some calculations [5, 6, 7, show that the real part of the $K^{-}$-nucleus optical potential $U_{K^{-}}$ is shallow $\left(U_{K^{-}} \approx-50 \mathrm{MeV}\right)$, but some other calculations suggest that $U_{K^{-}}$can be as large as about $-120 \mathrm{MeV}[8$, 9] or even close to $-200 \mathrm{MeV}$ [10]. We consider the possibility of deep optical potential of kaons in nuclei and explore the consequences in the composition of neutron star matter and the mass-radius relation of the neutron star.

To treat the dense nuclear matter we employ two different relativistic mean field models; the modified quarkmeson coupling (MQMC) model [11 and the quantum hadrodynamics (QHD) model [12. In the MQMC model the nucleons and hyperons in the baryon octet are treated as MIT bags, whereas in QHD they are assumed to be point particles. The parameters of the two models are calibrated to produce exactly the same saturation properties. By comparing the results from the two different models we can investigate the model dependence at high densities. We find the onset densities of the kaon condensation and the compositions of matter at high densities are nonnegligibly model dependent. The mass-radius relation of the neutron star can significantly differ depending on the interaction of the kaon in nuclear matter.

\section{Models}

The model Lagrangian consists of several terms for the octet baryons, exchange mesons, leptons and kaons; $\mathcal{L}_{t o t}=$ $\mathcal{L}_{B}+\mathcal{L}_{M}+\mathcal{L}_{l}+\mathcal{L}_{K}$. In the mean-field approximation, each term reads

$$
\begin{aligned}
\mathcal{L}_{B}= & \sum_{B} \bar{\psi}_{B}\left[i \gamma \cdot \partial-m_{B}^{*}\left(\sigma, \sigma^{*}\right)\right. \\
& \left.-\gamma^{0}\left(g_{\omega B} \omega_{0}+g_{\phi B} \phi_{0}+\frac{1}{2} g_{\rho B} \tau_{z} \rho_{03}\right)\right] \psi_{B}, \\
\mathcal{L}_{M}= & -\frac{1}{2} m_{\sigma}^{2} \sigma^{2}-\frac{1}{2} m_{\sigma^{*}}^{2} \sigma^{* 2}+\frac{1}{2} m_{\omega}^{2} \omega_{0}^{2}+\frac{1}{2} m_{\phi}^{2} \phi_{0}^{2} \\
& +\frac{1}{2} m_{\rho}^{2} \rho_{03}^{2}, \\
\mathcal{L}_{l}= & \sum_{l} \bar{\psi}_{l}\left(i \gamma \cdot \partial-m_{l}\right) \psi_{l}, \\
\mathcal{L}_{K}= & D_{\mu}^{*} K^{*} D^{\mu} K-m_{K}^{*}{ }^{2} K^{*} K,
\end{aligned}
$$

where $B$ denotes the sum over the octet baryons and $l$ stands for the sum over the free electrons and muons. The interactions between the non-strange light quarks ( $u$ and 
S. W. Hong et al.: Kaon condensation in neutron star using relativistic mean field models

$d$ ) are mediated by $\sigma, \omega$ and $\rho$ mesons, and $\sigma^{*}$ and $\phi$ mesons are introduced to take into account the interactions between $s$ quarks. One of the most important differences between MQMC and QHD lies in the treatment of baryons; the former treats baryons as composite systems of quarks, and the latter assumes baryons as point particles. As a result, the analytic form of the effective mass of a baryon differs greatly as follows.

In MQMC, the effective mass of a baryon in matter $m_{B}^{*}\left(\sigma, \sigma^{*}\right)$ can be written as $m_{B}^{*}=\sqrt{E_{B}^{2}-\sum_{q}\left(\frac{x_{q}}{R}\right)^{2}}$, where $E_{B}$ is the bag energy of a baryon, $R$ the bag radius and $x_{q}$ the eigenvalue of the quarks in the bag. The bag energy of a baryon is given by $E_{B}=\sum_{q} \frac{\Omega_{q}}{R}-\frac{Z_{B}}{R}+\frac{4}{3} \pi R^{3} B_{B}$, where $B_{B}$ and $Z_{B}$ are the bag constant and a phenomenological constant for the zero-point motion of a baryon $B$, respectively, and $\Omega_{q}=\sqrt{x_{q}^{2}+\left(R m_{q}^{*}\right)^{2}}$, where $m_{q}^{*}(=$ $\left.m_{q}-g_{\sigma}^{q} \sigma-g_{\sigma^{*}}^{q} \sigma^{*}\right)$ is the effective mass of a quark. In the MQMC model, the bag constant $B_{B}$ is assumed to depend on density, and we use the extended form of Ref. [3].

In QHD, the effective mass of a baryon is written as $m_{B}^{*}=m_{B}-g_{\sigma B} \sigma-g_{\sigma^{*} B} \sigma^{*}$. We require MQMC and QHD to satisfy identically the same saturation properties. To produce the compression modulus within a reasonable range one needs to include self-interaction terms of the $\sigma$-field represented by $U_{\sigma}^{\mathrm{QHD}}=\frac{1}{3} g_{2} \sigma^{3}+\frac{1}{4} g_{3} \sigma^{4}$ in the Lagrangian for QHD. The meson term in the QHD thus reads $\mathcal{L}_{M}^{\mathrm{QHD}}=\mathcal{L}_{M}-U_{\sigma}^{\mathrm{QHD}}$.

The kaon is treated as a point particle in both MQMC and QHD models in this work, and its effective mass is given as $m_{K}^{*}=m_{K}-g_{\sigma K} \sigma-g_{\sigma^{*} K} \sigma^{*}$. Covariant derivatives in Eq. (4) include interactions with vector mesons through $D_{\mu}=\partial_{\mu}+i g_{\omega K} \omega_{\mu}-i g_{\phi K} \phi_{\mu}+i \frac{1}{2} g_{\rho K} \boldsymbol{\tau} \cdot \boldsymbol{\rho}_{\mu}$. A plane wave solution for the kaon field equation gives the dispersion relation of the anti-kaon

$$
\omega_{K}=m_{K}^{*}-g_{\omega K} \omega_{0}+g_{\phi K} \phi_{0}-g_{\rho K} \frac{1}{2} \rho_{03}
$$

Nuclear saturation properties are relatively well known, and the values quoted in the literatures lie in the range $0.15 \sim 0.17 \mathrm{fm}^{-3}$ for the saturation density $\rho_{0}, 15 \sim 16.3$ $\mathrm{MeV}$ for the binding energy per nucleon $E_{b}, 30 \sim 35 \mathrm{MeV}$ for the symmetry energy $a_{\text {sym }}, 200 \sim 300 \mathrm{MeV}$ for the compression modulus $K$, and $(0.7 \sim 0.8) m_{N}$ for the effective mass of the nucleon. In this work we assume the saturation properties $\rho_{0}=0.17 \mathrm{fm}^{-3}, E_{b}=16.0 \mathrm{MeV}$, $a_{\text {sym }}=32.5 \mathrm{MeV}, K=285 \mathrm{MeV}$, and $m_{N}^{*}=0.78 m_{N}$. These conditions determine the coupling constants of nonstrange mesons to $u$ and $d$ quarks in the MQMC, and to nucleons in the QHD. These coupling constants are summarized in Table 1

For the coupling constants between the non-strange mesons $(\sigma, \omega$, and $\rho)$ and hyperons we use the quark counting rule assuming that the $s$-quark does not couple to $u$ and $d$ quarks. This gives us $g_{\sigma}^{s}=g_{\omega}^{s}=g_{\rho}^{s}=0$, and these relations determine the coupling constants of non-strange mesons to hyperons in both MQMC and QHD. For the coupling constants of strange meson to hyperons, we as-
Table 1. Non-strange meson coupling constants for the MQMC (left four columns) and for the QHD (right four columns) models. $g_{\rho N}$ in QHD is the same as $g_{\rho}^{q}$ in MQMC.

\begin{tabular}{cccc|cccc}
\hline$g_{\sigma}^{q}$ & $g_{\omega}^{q}$ & $g_{\sigma}^{\prime B}$ & $g_{\rho}^{q}$ & $g_{\sigma N}$ & $g_{\omega N}$ & $g_{2}\left(\mathrm{fm}^{-1}\right)$ & $g_{3}$ \\
\hline 1.0 & 2.71 & 2.27 & 7.88 & 8.06 & 8.19 & 12.1 & 48.4 \\
\hline
\end{tabular}

sume $\mathrm{SU}(6)$ symmetry, which gives us $g_{\sigma^{*}}^{s}=\sqrt{2} g_{\sigma}^{u, d}$ and $g_{\phi}^{s}=\sqrt{2} g_{\omega}^{u, d}$.

We have five meson-kaon coupling constants, $g_{\sigma K}, g_{\omega K}$, $g_{\rho K}, g_{\sigma^{*} K}$ and $g_{\phi K}$. The quark counting rule is employed for $g_{\omega K}$ and $g_{\rho K} \cdot g_{\sigma^{*} K}$ can be fixed from $f_{0}(980)$ decay, and $g_{\phi K}$ from the $\mathrm{SU}(6)$ relation $\sqrt{2} g_{\phi K}=g_{\pi \pi \rho} . g_{\sigma^{*} K}$ and $g_{\phi K}$ thus fixed are 2.65 and 4.27 , respectively. The remaining coupling constant, $g_{\sigma K}$, can be related to the real part of the optical potential of a kaon at the saturation density through $U_{K^{-}}=-\left(g_{\sigma K} \sigma+g_{\omega K} \omega_{0}\right)$. The resulting $g_{\sigma K}$ values for several $U_{K^{-}}$are given in Table 2.

Once the coupling constants are determined, one can obtain the EoS of neutron star matter by solving selfconsistently the equation of motion of exchange mesons, charge neutrality condition, baryon number conservation, and $\beta$-equilibrium conditions for baryons and kaons. The mass-radius relation of the neutron star can be obtained by inserting the EoS into the Tolman-Oppenheimer-Volkoff (TOV) equation.

\section{Results}

A particle fraction is defined by the density of a particle divided by the baryon density. Numerical results of the particle fraction is shown in Fig. 1. The onset density of the kaon condensation $\rho_{\text {crit }}$ decreases as $\left|U_{K^{-}}\right|$increases. This is expected because a larger $g_{\sigma K}$ makes $m_{K}^{*}$ smaller, which then causes the chemical equilibrium of kaons, $\omega_{K}=\mu_{n}-\mu_{p}$ fulfilled at lower densities, where $\mu_{n(p)}$ is the chemical potential of the neutron (proton). An interestring feature is that once the kaon is created, the density of $K^{-}$increases very rapidly and dominates the particle population over the hyperons and even the nucleons. This can be partly attributed to the fact that the contribution from the $\omega$-meson to the octet baryons is repulsive whereas the contribution from the $\omega$-meson to the kaon is attractive. The $\omega$-meson term in the energy of $K^{-}$in Eq. (5) has a negative sign and is thus attractive, but it is repulsive for the chemical potential of the octet baryons. The $\omega$-meson enhances the population

Table 2. $g_{\sigma K}$ for several $U_{K^{-}}$values in MQMC and QHD.

\begin{tabular}{cccc}
\hline$U_{K^{-}}(\mathrm{MeV})$ & -120 & -140 & -160 \\
\hline MQMC & 2.75 & 3.50 & 4.25 \\
QHD & 2.83 & 3.61 & 4.39 \\
\hline
\end{tabular}



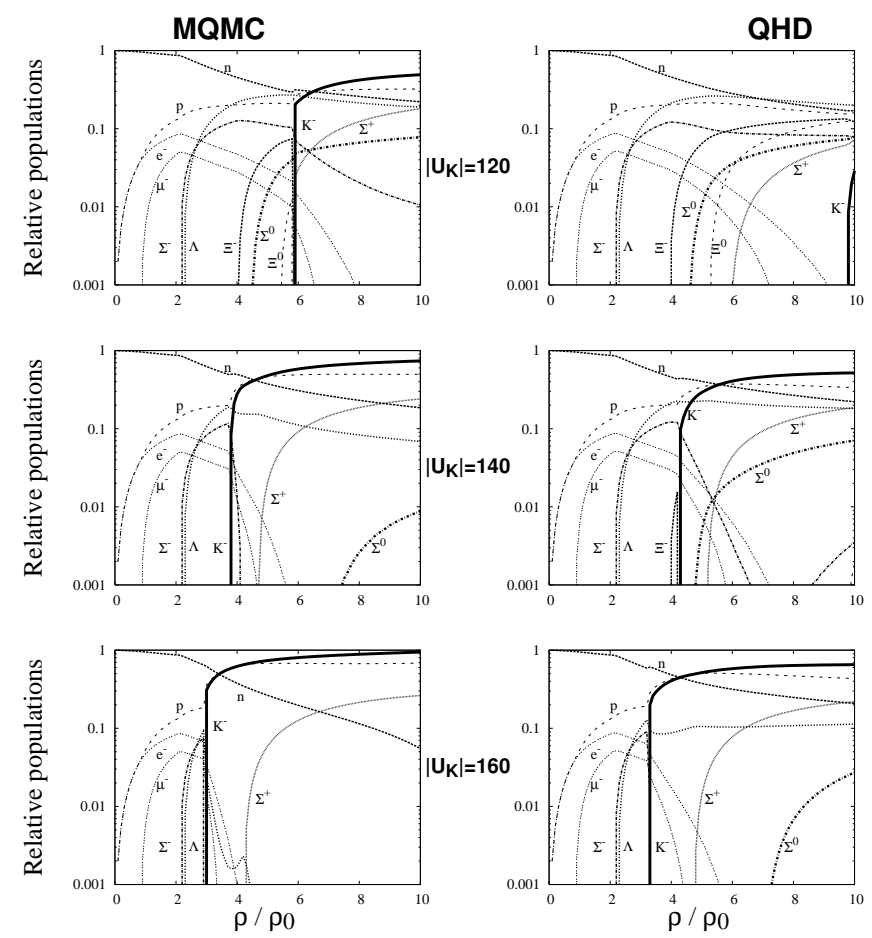

Fig. 1. The relative populations of particles in nuclear matter calculated from the MQMC and QHD models are displayed in the left and right columns, respectively, for $U_{K^{-}}=-120$, -140 and $-160 \mathrm{MeV}$.

of $K^{-}$but suppresses baryons, and thus the kaon density increases rapidly. In addition, due to the competition between the negatively charged hyperons and $K^{-}$in the charge neutrality condition, the negatively charged hyperons are highly suppressed and in some cases are not even created at all as soon as the kaon condensation sets in. Positively charged hyperons, on the other hand, receive the opposite effects from the kaon condensation, and $\Sigma^{+}$ is created at lower densities as $\left|U_{K^{-}}\right|$increases. The proton density is also enhanced by large abundance of $K^{-}$, which facilitates in turn the enhancement of $\Sigma^{+}$population through the chemical equilibrium condition of the positively charged hyperons.

We find that the onset density of kaon condensation $\rho_{\text {crit }}$ from the MQMC model is lower than that from QHD. For $U_{K^{-}}=-120,-140$, and $-160 \mathrm{MeV}, \rho_{\text {crit }}$ from MQMC are $5.9 \rho_{0}, 3.8 \rho_{0}$ and $3.0 \rho_{0}$, respectively, while they are $9.8 \rho_{0}, 4.3 \rho_{0}$ and $3.3 \rho_{0}$ in QHD. The model dependence of $\rho_{\text {crit }}$ becomes less pronounced for a larger $\left|U_{K^{-}}\right|$value.

Kaon condensation leads to a phase transition from a pure hadronic phase to a kaon condensed one with a mixed state of hadron and kaon phases. To deal with such a mixed phase one needs to use Gibbs conditions. However, for simplicity, we employ Maxwell condition for the mixed phase, which is often used as an approximation to the Gibbs condition. Though the Maxwell construction may not give us very accurate results particularly for the EoS, it can still provdie us with reasonable results for the mass-radius relation of the neutron star.
Fig. 2 shows the EoS including kaon condensation, but calculated without using either Maxwell or Gibbs condition. There is an instability region where pressure decreases as the density increases. If such an instability exists in the interior of a neutron star, there is a high pressure region on top of a low pressure one. The low pressure region cannot sustain the high pressure region, and the unstable region will collapse, and the matter will be rearranged to fix the instability. Including such a rearrangement in the calculation of the EoS is equivalent to implmenting Maxwell or Gibbs condition. With Maxwell condition, the unstable part is replaced by a flat line, which is determined by the equal area method. (The flat lines are not plotted in Fig. 2 to avoid too many lines in one figure but should be obvious.) From Fig. 2 we can see that kaon condensation begins at a point where the EoS curve deviates from the solid curve obtained without kaons. The kaon condensation makes the EoS softer than the one without it. As $\left|U_{K^{-}}\right|$becomes larger, the EoS becomes softer. By comparing the two solid curves in the left and in the right panels we see that the EoS without kaons is softer with the QHD model, but the effect of kaon condensation is more significant in the MQMC model.

Once the EoS is determined, it is straightforward to solve the TOV equation to obtain the mass-radius relation of a neutron star. Fig. 3 shows the mass-radius curves calculated by the MQMC (left) and QHD (right) models. For $U_{K^{-}}=-120 \mathrm{MeV}$, the effect of kaon condensation is rather small and negligible particularly for the QHD case. The maximum mass of a neutron star with only nucleons is about $2 M_{\odot}$, as mentioned in the Introduction. When hyperons are created, the maximum mass
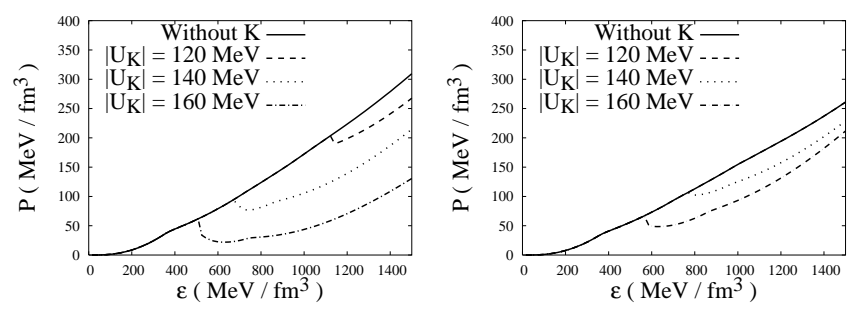

Fig. 2. The EoS without using any equilibrium condition in the mixed phase. The left figure is from MQMC, and the right figure is from QHD. The unstable region where the pressure decreases with increasing density has to be remedied by either Maxwell or Gibbs condition.
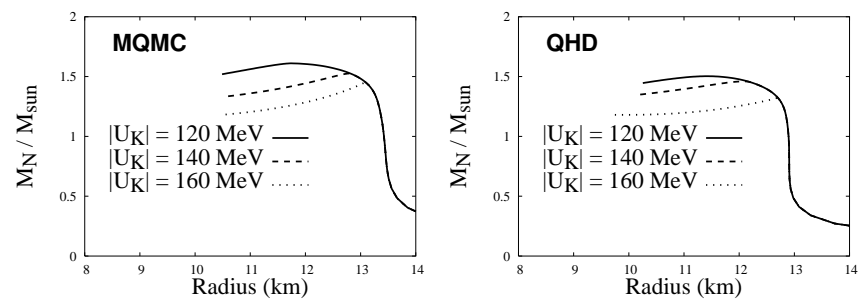

Fig. 3. The mass-radius relation from the MQMC (left) and QHD (right) models. Both figures are obtained with Maxwell construction. 

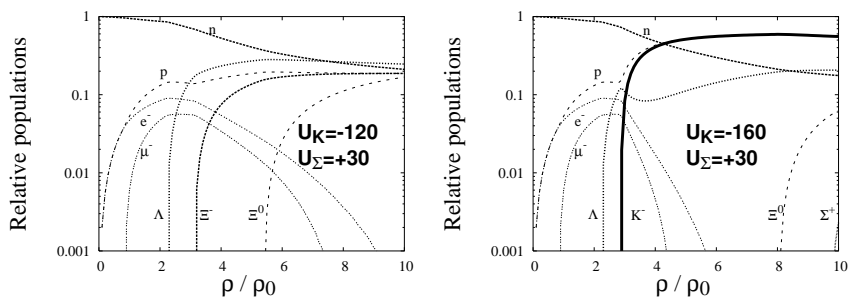

Fig. 4. The particle fraction with repulsive in-medium interaction of $\Sigma$ hyperon in the QHD model. $U_{\Sigma}$ is fixed as +30 $\mathrm{MeV}$, and $U_{K^{-}}$is chosen as -120 (left) and -160 (right) $\mathrm{MeV}$.

of a neutron star decreases about $0.5 M_{\odot}$. As the depth of optical potential for $K^{-}$increases, the effect of kaon condensation becomes more significant. If $U_{K^{-}}=-160$ $\mathrm{MeV}$, the maximum mass decreases by about $10 \sim 12 \%$ from the maximum mass without kaon condensation. In the case of QHD the maximum mass with $U_{K^{-}}=-160$ $\mathrm{MeV}$ becomes so small as $1.32 M_{\odot}$ that most of the observed masses in Ref. [1] exceeds this (maximum) value. This raises a question whether a very deep optical potential of $K^{-}$can be comptible with the existing observations of the neutron star mass. We will discuss this again in the next section.

One of the unsettled issues in hypernuclear physics is the interaction of $\Sigma$ hyperon in nuclear medium. Use of plain quark counting rule for the hyperon-meson coupling constants gives us attractive optical potentials of $\Sigma$ hyperons in the range $-40 \sim-30 \mathrm{MeV}$ at the saturation density. Some studies of $\Sigma$-hypernuclei, however, indicates that the interaction of $\Sigma$ hyperon in medium is repulsive and its optical potential at the saturation density takes a positive value [13. To take into account this possibility, we have repeated the calculations using the QHD model with the repulsive $\Sigma$-hyperon optical potential of $+30 \mathrm{MeV}$ at the saturation density and show the

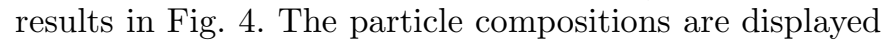
for $U_{K^{-}}=-120$ and $-160 \mathrm{MeV}$. By comparing these two figures with the figures on the right panels of Fig. 1 one can see that the repulsive $\Sigma$-hyperon interaction makes big changes in the population of hyperons, but the onset densities and the population of kaons are little affected. The maximum masses of a neutron star with $U_{\Sigma}=+30$ $\mathrm{MeV}$ are $1.52 M_{\odot}, 1.48 M_{\odot}$ and $1.32 M_{\odot}$ for $U_{K^{-}}=-120$, -140 and $-160 \mathrm{MeV}$, respectively. These results deviate from those with attractive $\Sigma$-hyperon interaction only by about $1.4 \%$ at most.

\section{Discussion}

Masses of some pulsars [14,15, that are more recently measured are reported to lie much above the canonical value of Ref. 11. Some of the large masses are in the range of $(1.7-2.1) M_{\odot}$, though the results depend on the binary systems. These values are even larger than the maximum mass of a neutron star composed of only nucleons and hyperons (in short, a baryon star). If kaon condensate states are added to the baryonic state, it will soften the EoS and reduce the maximum mass further down. Then one confronts the question: Are exotic states such as kaon condensation ruled out by large mass neutron stars? To answer the question, we have to understand better the interaction of particles and state of matter at densities above the nuclear saturation. For instance, we don't know yet to what densities our mean field lagrangian models are valid. If the large mass neutron star does exist, we have to have a mechanism which makes the EoS at high densities stiffer than now. Introducing hard core repulsion between nucleons can make the EoS stiff at high densities. The effect of hard core has been considered in the high density/temperature limit region [16,17,18, and it is shown that it makes the deconfined quark phase the most stable state of matter at high density and/or temperature. One can take into account such effects in the neutron star matter together with exotic states. The investigation is in progress.

This work was supported by grant No.R01-2005-00010050-0 from the Basic Research Program of the Korea Science \& Engineering Foundation. Work of CHH was supported by Korea Research Foundation Grant funded by Korea Government (MOEHRD, Basic Research Promotion Fund) (KRF-2005-206-C00007).

\section{References}

1. S. E. Thorsett and D. Chakrabarty, Astrophys. J. 512, 288 (1999).

2. R. Knorren, M. Prakash and P. J. Ellis, Phys. Rev. C 52, 3470 (1995).

3. S. Pal, M. Hanauske, I. Zakout, H. Stöcker and W. Greiner, Phys. Rev. C 60, 015802 (1999).

4. N. K. Glendenning, Phys. Rev. D 46, 1274 (1992).

5. J. Schaffner-Bielich, V. Koch and M. Effenberger, Nucl. Phys. A669, 153 (2000).

6. A. Ramos and E. Oset, Nucl. Phys. A671, 481 (2000).

7. A. Cieply, E. Friedman, A. Gal and J. Mares, Nucl. Phys. A696, 173 (2001).

8. E. Friedman, A. Gal and C. J. Batty, Nucl. Phys. A579, 578 (1994).

9. N. Kaiser, P.B. Siegel and W. Weise, Nucl. Phys. A594, 325 (1995).

10. C. J. Batty, E. Friedman, A. Gal, Phys. Rep. 287, 385 (1997).

11. X. Jin and B. K. Jennings, Phys. Lett. B374, 13 (1996); Phys. Rev. C 54, 1427 (1996).

12. B. D. Serot and J. D. Walecka, Adv. Nucl. Phys. 16, 1 (1986).

13. P. K. Saha et al., Phys. Rev. C 70, 044613 (2004).

14. D. J. Nice et al., Astrophys. J. 634, 1242 (2005).

15. D. Page and S. Reddy, Ann. Rev. Nucl. Part. Sci. 56, 327 (2006).

16. D. H. Rischke, M. I. Gorenstein, H. Stöcker and W. Greiner, Z. Phys. C 51, 485 (1991).

17. S. Kagiyama, A. Nakamura and T. Omodaka, Z. Phys. C 53, 163 (1992).

18. J. Cleymans, J. Stalnacke and E. Suhonen, Z. Phys. C 55, 317 (1992). 
